\title{
Safe glueballs and baryons
}

\section{Thomas A. Ryttov ${ }^{a}$ and Kimmo Tuominen ${ }^{b}$}

${ }^{a} C P^{3}$-Origins, University of Southern Denmark, Campusvej 55, Odense M, 5230 Denmark

${ }^{b}$ Department of Physics $\&$ Helsinki Institute of Physics, P.O. Box 64, University of Helsinki, Helsinki, FI-00014 Finland

E-mail: ryttov@cp3.sdu.dk, kimmo.i.tuominen@helsinki.fi

ABSTRACT: We consider a non-Abelian gauge theory with $N_{f}$ fermions and discuss the possible existence of a non-trivial UV fixed point at large $N_{f}$. Specifically, we study the anomalous dimension of the (rescaled) glueball operator $\operatorname{Tr} F^{2}$ to first order in $1 / N_{f}$ by relating it to the derivative of the beta function at the fixed point. At the fixed point the anomalous dimension violates its unitarity bound and so the (rescaled) glueball operator is either decoupled or the fixed point does not exist. We also study the anomalous dimensions of the two spin- $1 / 2$ baryon operators to first order in $1 / N_{f}$ for an $\mathrm{SU}(3)$ gauge theory with fundamental fermions and find them to be relatively small and well within their associated unitarity bounds.

Keywords: 1/N Expansion, Lattice Quantum Field Theory, Renormalization Group

ArXiv ePrint: 1903.09089 


\section{Contents}

1 Introduction 1

2 The beta function at large $N_{f} \quad 2$

3 Dimensions of operators at large $N_{f} \quad 3$

3.1 Dimension of (rescaled) glueball operator $\operatorname{Tr} F^{2}$

3.2 Dimension of spin- $1 / 2$ baryon operators $\psi \psi \psi$ in an $\mathrm{SU}(3)$ gauge theory 5

4 Dimensions at the UV fixed point at large $N_{f} \quad 6$

5 Conclusion $\quad 9$

$\begin{array}{lr}\text { A Beta function coefficients } & 10\end{array}$

B The functions $\Gamma(z), \psi(z), \psi^{(m)}(z)$ and $\zeta(z) \quad 11$

\section{Introduction}

Gauge theory with many flavors of fermions may possess an ultraviolet (UV) fixed point. The daunting task of computing specific renormalization group functions for gauge theories at large number of fermions was first undertaken in Abelian gauge theory in [1] and in the non-Abelian case in [2]. Recently, there has been a revived interest in asymptotically safe four-dimensional quantum field theories, with particular emphasis on pure fermionic gauge theories, chiral gauge theories, gauge-Yukawa theories involving both scalars and fermions and theories with or without supersymmetry [3-21]. For a recent review of the technical and computational aspects see [22].

The earlier works [23] on fermionic gauge theory phase diagrams as a function of the number of colors, fermion flavors and their representations were extended to address also the domain where a UV fixed point might emerge in [9]. While the studies of an infrared (IR) fixed point have been studied both analytically and numerically, the corresponding methodology to study UV fixed points is currently developing. The work reported in this paper is part of this ongoing effort to elucidate the phase structure of gauge theories at large $N_{f}$ and should be contrasted to other analytical calculations as well as future lattice simulations.

In this paper we will first review the results on the exact gauge theory beta function to leading order in $1 / N_{f}$. Our main results are the formulas for the anomalous dimensions of the glueball operator and for the two spin- $1 / 2$ baryon operators to leading order in $1 / N_{f}$. We will then determine the location of the UV fixed point and evaluate the anomalous dimensions at the fixed point. 
We find that while the anomalous dimensions of the baryon operators have small values at the fixed point, well consistent with the unitarity bound, the anomalous dimension of the glueball operator grows rapidly with increasing $N_{f}$ grossly violating its unitarity bound. We will discuss the interpretation of this result. One possible interpretation is that the leading order beta function is inconsistent and there is no fixed point. However, an equally viable interpretation is that the glueball operator is decoupled and not part of the spectrum.

The paper is organized as follows: in section 2 we present the leading order beta function in detail. Then, in section 3 we derive the general results for the operator dimensions of the glueball operator and baryon spin-1/2 operators, and in section 4 we analyze these operator dimensions at a UV fixed point. In section 5 we present our conclusions and outlook for further work.

\section{The beta function at large $N_{f}$}

We consider a fermionic gauge theory with gauge group $G$ and $N_{f}$ number of Dirac fermions in some representation $r$ of $G$. We let $T_{r}$ denote the trace normalization factor and $C_{r}$ the quadratic Casimir of the generators in the representation $r$ while $A$ denotes the adjoint representation. As an expansion in the gauge coupling $\alpha=\frac{g^{2}}{4 \pi}$ we write the beta function as

$$
\beta(\alpha)=\mu \frac{d \alpha}{d \mu}=-\sum_{i=1}^{\infty} b_{i} \frac{\alpha^{i+1}}{\pi^{i}}=-b_{1} \frac{\alpha^{2}}{\pi}-b_{2} \frac{\alpha^{3}}{\pi^{2}}-b_{3} \frac{\alpha^{4}}{\pi^{3}}-\ldots
$$

Within the class of mass independent schemes the first two coefficients are universal whereas from higher orders they become scheme dependent. Most recently the five loop coefficient of the beta function has been computed in the $\overline{\mathrm{MS}}$ scheme $[24,25]$ which now represents the highest loop calculation for non-Abelian gauge theories. These coefficients generally depend on various (higher order) group invariants, the Riemann zeta function $\zeta_{s}$ and rational numbers.

All coefficients $b_{i}, i \geq 1$ are polynomials in $N_{f}$. The first coefficient $b_{1}$ is a polynomial in $N_{f}$ to $\mathcal{O}\left(N_{f}\right)$ while all the remaining coefficients $b_{i}, i \geq 2$ are polynomials in $N_{f}$ to order $\mathcal{O}\left(N_{f}^{i-1}\right)$. For these higher order coefficients we will then write

$$
b_{i}=b_{i, 0}+b_{i, 1} N_{f}+\ldots+b_{i, i-1} N_{f}^{i-1}=\sum_{j=0}^{i-1} b_{i, j} N_{f}^{j}, \quad i \geq 2 .
$$

Instead of expanding in the gauge coupling $\alpha$ it is possible to reformulate perturbation theory as an expansion in $1 / N_{f}$ with the normalized coupling $A \equiv T_{r} N_{f} \frac{\alpha}{\pi}$ held fixed [1-3]. Here one rewrites the beta function of the gauge coupling by switching to the normalized coupling $A \equiv T_{r} N_{f} \frac{\alpha}{\pi}$ and then collecting terms in powers of $1 / N_{f}$. One can then study the theory in terms of perturbation theory in $1 / N_{f}$. Changing variables, we find that the beta function can instead be written as

$$
\beta(A)=\mu \frac{d A}{d \mu}=\frac{2}{3} A^{2}\left(1+\sum_{i=1}^{\infty} \frac{H_{i}(A)}{N_{f}^{i}}\right) .
$$


The functions $H_{i}(A)$ depend on the scaled coupling $A$ and are directly related to the coefficients of the original beta function. Explicitly, they are given by

$$
\begin{aligned}
& H_{1}(A)=-\frac{11}{4} \frac{C_{A}}{T_{r}}-\frac{3}{2} \sum_{j=2}^{\infty} \frac{b_{j, j-1}}{T_{r}^{j}} A^{j-1}, \\
& H_{i}(A)=-\frac{3}{2} \sum_{j=i}^{\infty} \frac{b_{j, j-i}}{T_{r}^{j}} A^{j-1}, \quad i \geq 2 .
\end{aligned}
$$

Remarkably, the first term $H_{1}(A)$ has been calculated to all orders in $A$ so that the beta function is known exactly to first non-trivial order in $1 / N_{f}$. It was calculated for QED in [1] while for QCD it was calculated in [2]. Using the notation of [3] it is explicitly given by

$$
\begin{aligned}
H_{1}(A) & =-\frac{11}{4} \frac{C_{A}}{T_{r}}+\int_{0}^{\frac{A}{3}} I_{1}(x) I_{2}(x) d x \\
I_{1}(x) & =\frac{(1+x)(2 x-1)^{2}(2 x-3)^{2} \sin (\pi x)^{3} \Gamma(x-1)^{2} \Gamma(-2 x)}{(x-2) \pi^{3}}, \\
I_{2}(x) & =\frac{C_{r}}{T_{r}}+\frac{\left(20-43 x+32 x^{2}-14 x^{3}+4 x^{4}\right)}{4(2 x-1)(2 x-3)(1+x)(1-x)} \frac{C_{A}}{T_{r}}
\end{aligned}
$$

In the left panel of figure 1 the beta function for fundamental matter and $N_{c}=3$ to first order in $1 / N_{f}$ is shown for a range of values of $N_{f}$. In the right panel of figure 1 we show the comparison of the beta function to leading order in $1 / N_{f}$ and the perturbative beta function at different loop orders for $N_{f}=100$. Interestingly, the leading order beta function and five loop beta function agree very well almost up to $A=3$ where the leading order beta function diverges.

At higher orders the functions $H_{i}(A)$ are not known exactly except for the first few orders in $A$. Specifically the first few terms in $H_{2}(A), \ldots, H_{5}(A)$ can be inferred from the five-loop beta function and we give explicit expressions for a general gauge group and fermion representation in appendix A. Useful relations between the Euler $\Gamma(z)$, the digamma function $\psi(z)$, the polygamma function $\psi^{(m)}(z)$ and the Riemann zeta function $\zeta(z)$ are provided in appendix B.

\section{Dimensions of operators at large $N_{f}$}

Of critical importance are the scaling dimensions of gauge invariant operators. At a fixed point they are scheme independent physical quantities. For some gauge invariant operator $O$ we shall in general denote its full scaling dimension by $D=d-\gamma$, where $d$ is its classical scaling dimension and $\gamma$ its anomalous dimension. Note that our convention of the minus sign if front of the anomalous dimension here. Dimensions of gauge invariant operators have a conventional expansion in the coupling as

$$
\gamma=\sum_{i=1}^{\infty} \gamma_{i} \frac{\alpha^{i}}{\pi^{i}} .
$$



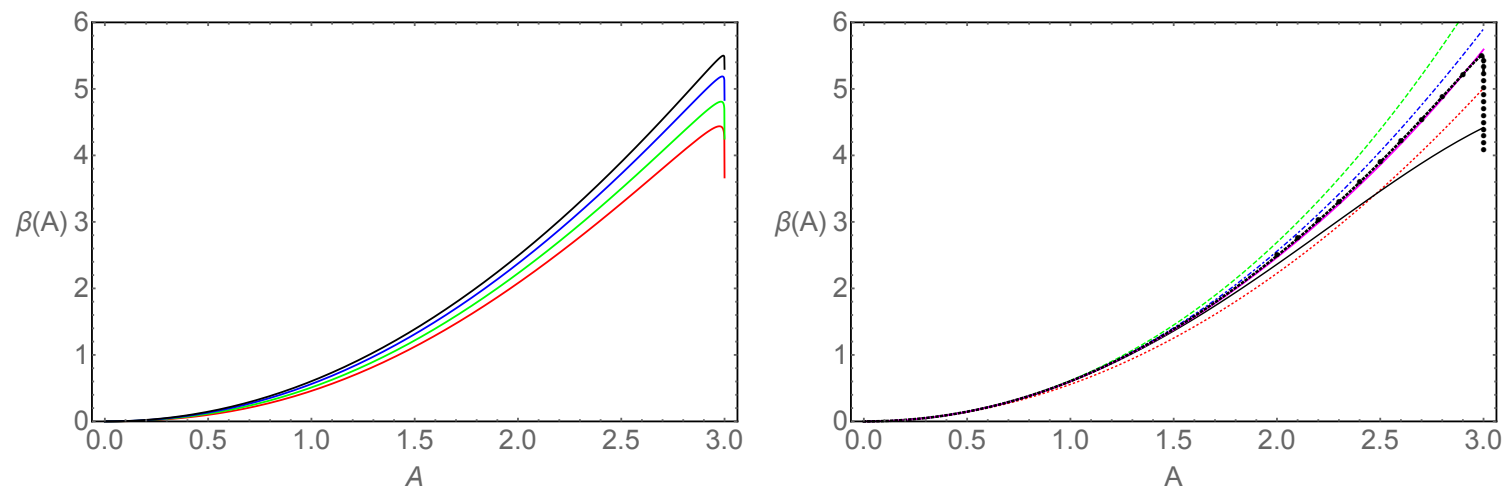

Figure 1. Left: the beta function to first order in $1 / N_{f}$ for matter fields in the fundamental representation and $N_{c}=3$. From bottom to top the curves are for $N_{f}=30$ (red), $N_{f}=40$ (green), $N_{f}=60$ (blue) and $N_{f}=100$ (black). Right: the beta function at different loop orders of perturbation theory for $N_{f}=100$. The dotted (red), dashed (green), dash-dotted (blue), solid (black) and thick solid (magenta) lines correspond, respectively to one, two, three, four and five loop results. The thick-dotted line is the beta function to leading order in $1 / N_{f}$.

Similar to the beta function coefficients the coefficients $\gamma_{i}$ of the anomalous dimension will in general be a polynomial in the number of flavors $N_{f}$. Therefore it is also possible to rearrange the perturbative expansion into a $1 / N_{f}$ expansion for the anomalous dimension. In general all of the coefficients of the anomalous dimension can be written as

$$
\gamma_{i}=\gamma_{i, 0}+\gamma_{i, 1} N_{f} \ldots+\gamma_{i, i-1} N_{f}^{i-1}=\sum_{j=0}^{i-1} \gamma_{i, j} N_{f}^{j}, \quad i \geq 1 .
$$

Note that the first coefficient $\gamma_{1}$ does not depend on $N_{f}$ which is different from the first coefficient of the beta function $b_{1}$. Now again changing variables to the normalized coupling $A=T_{r} N_{f} \frac{\alpha}{\pi}$ and collecting terms in powers of $1 / N_{f}$ we can instead write the anomalous dimension as

$$
\gamma=\sum_{i=1}^{\infty} \frac{G_{i}(A)}{N_{f}^{i}}
$$

where the functions $G_{i}(A)$ depend on $A$ and are directly given in terms of the original coefficients of the anomalous dimension as

$$
G_{i}(A)=\sum_{j=1}^{\infty} \frac{\gamma_{j, j-i}}{T_{r}^{j}} A^{j} .
$$

We will now determine the anomalous dimensions explicitly for the glueball and spin- $1 / 2$ baryon operators.

\subsection{Dimension of (rescaled) glueball operator $\operatorname{Tr} F^{2}$}

If the gauge field is denoted by $A_{\mu}^{a}$ then the canonical field strength tensor is $F_{\mu \nu}^{a}=\partial_{\mu} A_{\nu}^{a}-$ $\partial_{\nu} A_{\mu}^{a}+g f^{a b c} A_{\mu}^{b} A_{\nu}^{c}$. If we rescale the canonical gauge fields as $A_{\mu}^{a} \rightarrow \frac{1}{g} A_{\mu}^{a}$ then the canonical 
field strength tensor is rescaled as $F_{\mu \nu}^{a} \rightarrow \frac{1}{g} F_{\mu \nu}^{a}$ and the canonical gauge boson kinetic and self interaction term in the Lagrangian is rescaled as $-\frac{1}{4} F_{\mu \nu}^{a} F^{\mu \nu, a} \rightarrow-\frac{1}{4 g^{2}} F_{\mu \nu}^{a} F^{\mu \nu, a}$. From now on $F_{\mu \nu}^{a}$ will denote the field strength tensor rescaled in this way.

The dimension of the $F_{\mu \nu}^{a} F^{\mu \nu, a}$ operator is of great importance. First, we will show how the dimension of this operator is related to the beta function and its derivative. In order to do this we consider the trace anomaly

$$
T_{\mu}^{\mu}=\frac{\beta(\alpha)}{16 \pi \alpha^{2}} F_{\mu \nu}^{a} F^{\mu \nu, a}
$$

Taking the derivative on both sides of the trace anomaly by $\mu \frac{d}{d \mu}$ and using the fact that $T^{\mu}{ }_{\mu}$ must scale classically we find that the dimension of the $F_{\mu \nu}^{a} F^{\mu \nu, a}$ operator must be given by

$$
D_{F^{2}}=4+\beta^{\prime}(\alpha)-\frac{2 \beta(\alpha)}{\alpha}, \quad \gamma_{F^{2}}=-\beta^{\prime}(\alpha)+\frac{2 \beta(\alpha)}{\alpha}=\sum_{i=1}^{\infty}(i-1) b_{i} \frac{\alpha^{i}}{\pi^{i}},
$$

where $\beta^{\prime}(\alpha)=\frac{d \beta(\alpha)}{d \alpha}$ is the derivative of the beta function. So the dimension is given directly in terms of the beta function and its first derivative. The coefficients of the anomalous dimension are $\gamma_{i}=(i-1) b_{i}$. Note here that the first term $\gamma_{1}=0$ vanishes and so the first non-trivial contribution to the anomalous dimension begins at order $\alpha^{2}$.

Using eq. (2.3) and switching variable to $A$, we can instead write the dimension as an expansion in $1 / N_{f}$

$$
D_{F^{2}}=4+\sum_{i=1}^{\infty} \frac{\frac{2}{3} A^{2} H_{i}^{\prime}(A)}{N_{f}^{i}}, \quad \gamma_{F^{2}}=\sum_{i=1}^{\infty} \frac{-\frac{2}{3} A^{2} H_{i}^{\prime}(A)}{N_{f}^{i}} .
$$

This is one of our main results. It gives the dimension of the rescaled $F_{\mu \nu}^{a} F^{\mu \nu, a}$ operator to all orders in the coupling and first order in $1 / N_{f}$ since we know $H_{1}^{\prime}(A)$ from above. It corresponds to the summation of an infinite set of Feynman diagrams. Explicitly the anomalous dimension to first order in $1 / N_{f}$ is

$$
\gamma_{F^{2}}=-\frac{2}{9} A^{2} I_{1}(A / 3) I_{2}(A / 3) \frac{1}{N_{f}}+\mathcal{O}\left(1 / N_{f}^{2}\right)
$$

where $I_{1}$ and $I_{2}$ are given in eqs. (2.7)-(2.8).

\subsection{Dimension of spin-1/2 baryon operators $\psi \psi \psi$ in an $\mathrm{SU}(3)$ gauge theory}

The second set of operators which we will consider is constituted by the spin- $1 / 2$ baryon operators $\psi \psi \psi$ for an $\mathrm{SU}(3)$ gauge theory with $N_{f}$ fundamental flavors. Spin-1/2 baryon operators come in two types depending on how one contracts the Lorentz indices. In [26] the anomalous dimensions have been computed exactly to first order in $1 / N_{f}$ for both cases. Due to the presence of evanescent operators the anomalous dimensions contain a function which depends on the scheme in which it is calculated [26]. We write the full 
scaling dimension for each of these two operators as $D_{ \pm}=\frac{9}{2}-\gamma_{ \pm}$and using the results of [26] we find

$$
\gamma_{ \pm}=\frac{1}{2} \gamma_{m}\left(\frac{1}{2} A+\frac{s_{ \pm}(A)}{1-\frac{2}{9} A}\right)+\mathcal{O}\left(1 / N_{f}^{2}\right) .
$$

Here $s_{ \pm}(A)$ are the scheme dependent functions which depend on the coupling $A$ and satisfy $s_{ \pm}(0)=1$. We shall consider two different schemes adopted in [27, 28] for which $s_{ \pm}=1$ and $s_{ \pm}=\left(1-\frac{2}{9} A\right)\left(1-\frac{1}{6} A\right)$ respectively. Lastly $\gamma_{m}$ is the anomalous dimension of the mass and has been calculated exactly to second order in $1 / N_{f}$. It was calculated for QCD to first order in [1] and to second order in [29]. Here we only need it to first order for which it reads

$$
\gamma_{m}=\frac{C_{r}}{2 T_{r}} \frac{A\left(1-\frac{2}{9} A\right) \Gamma\left(4-\frac{2}{3} A\right)}{\Gamma\left(1+\frac{1}{3} A\right)\left[\Gamma\left(2-\frac{1}{3} A\right)\right]^{2} \Gamma\left(3-\frac{1}{3} A\right)} \frac{1}{N_{f}}+\mathcal{O}\left(1 / N_{f}^{2}\right),
$$

where $T_{r}=1 / 2$ and $C_{r}=4 / 3$ for fermions in the fundamental representation of SU(3). In ordinary perturbation theory it is known to five loop order in the $\overline{\mathrm{MS}}$ scheme [30, 31].

\section{Dimensions at the UV fixed point at large $N_{f}$}

The beta function has a trivial fixed point $(\alpha=0)$ which is in the IR provided the first coefficient is negative $b_{1}<0$, so that the one loop beta function is positive. For a given gauge group the theory is then infrared free if the number of flavors is sufficiently large, $N_{f}>\frac{11 C_{A}}{4 T_{r}}$. In this region of flavor space it is of considerable interest to ask what happens in the UV. For instance, does the theory run into a Landau pole (which is indicated by the one loop beta function) or does the theory develop a non-trivial UV fixed point. For sufficiently large $N_{f}$ there are indications that a new non-trivial fixed point is generated [3, 4, 9].

To understand if a non-trivial UV fixed point $A_{\mathrm{UV}}$ might be generated we need to study the possible zeros of the beta function. To first order in $1 / N_{f}$ we look for solutions to the equation

$$
1+\frac{H_{1}\left(A_{\mathrm{UV}}\right)}{N_{f}}=0
$$

At first sight this might not seem to have any solutions in the large $N_{f}$ limit. However there are singularities hiding in $H_{1}(A)$ that can balance $1 / N_{f}$ such as to make the ratio $H_{1}\left(A_{\mathrm{UV}}\right) / N_{f}$ finite and equal to -1 . Examining the integrand $I_{1}(x) I_{2}(x)$ of $H_{1}(A)$, we see that the first divergence for $x>0$ occurs at $x=1$. This stems from the $\frac{1}{1-x}$ term in $I_{2}(x)$ and hence gives rise to a logarithmic divergence in $H_{1}(A)$ at $x=1$. To be more explicit we now expand the integrand in $H_{1}(A)$ around $x=1$ in order to find

$$
I_{1}(x) I_{2}(x)=-\frac{C_{A}}{16 T_{r}} \frac{1}{1-x}+\sum_{i=0}^{\infty} \frac{a_{i} C_{A}+b_{i} C_{r}}{16 T_{r}}(1-x)^{i},
$$

where $a_{i}$ and $b_{i}$ are the expansion coefficients which are pure numbers not depending on any group factors. The first few are $a_{0}=3, a_{1}=23, a_{2}=29-\psi^{(2)}(1)$ and $b_{0}=-8, b_{1}=$ 
$-12, b_{2}=32$. This expansion then gives us $H_{1}(A)$ with the divergence isolated as a logarithm

$$
H_{1}(A)=\frac{C_{A}}{16 T_{r}}\left[\ln \left(1-\frac{A}{3}\right)-a-b \frac{C_{r}}{C_{A}}+\sum_{i=0}^{\infty}\left(a_{i}+b_{i} \frac{C_{r}}{C_{A}}\right) \frac{\left(1-\frac{A}{3}\right)^{i+1}}{i+1}\right],
$$

where we have defined $a=44-\sum_{i=0}^{\infty} \frac{a_{i}}{i+1}$ and $b=-\sum_{i=0}^{\infty} \frac{b_{i}}{i+1}$. These two constants cannot be calculated exactly, but using the first 20 expansion coefficients $a_{i}, b_{i}, i=0 \ldots, 20$ we find that numerically $a=17.39$ and $b=-5.26$. Both series seem to have safely converged to these values at this order.

We should then look for solutions to $H_{1}\left(A_{\mathrm{UV}}\right)=-N_{f}$ at large $N_{f}$. Clearly $H_{1}(A)$ diverges as $A \rightarrow 3$ due to the logarithm term. In this limit, all the terms to the right which are polynomial in $(3-A)$ can be neglected. The resulting equation can be solved for $A_{\mathrm{UV}}$, and we find

$$
A_{\mathrm{UV}}=3-\delta, \quad \delta=\exp \left[-16 \frac{T_{r}}{C_{A}} N_{f}+18.49-5.26 \frac{C_{r}}{C_{A}}\right] .
$$

This result is in agreement with the results in $[5,9]$. We will now assume that this fixed point exists for sufficiently large $N_{f}$ and study the anomalous dimensions of the glueball operator $\gamma_{F^{2}}$ and the spin-1/2 baryons $\gamma_{ \pm}$. At a fixed point the theory is scale invariant and dimensions of gauge invariant operators are scheme independent physical quantities.

The anomalous dimension of the $\operatorname{Tr} F^{2}$ operator at the UV fixed point in first order in $1 / N_{f}$ becomes

$$
\gamma_{F^{2}}=-\frac{2}{9} A_{\mathrm{UV}}^{2} I_{1}\left(A_{\mathrm{UV}} / 3\right) I_{2}\left(A_{\mathrm{UV}} / 3\right) \frac{1}{N_{f}}=\frac{3 C_{A}}{8 T_{r}} \frac{1}{N_{f}} \delta^{-1}+\mathcal{O}\left(\delta^{0}\right) .
$$

So the anomalous dimension increases exponentially in the large $N_{f}$ limit due to the $\delta^{-1}$ contribution. The anomalous dimension is positive (since the derivative of the beta function is negative) and therefore the full dimension $D_{F^{2}}=4-\gamma_{F^{2}}$ blows to minus infinity in the large $N_{c}$ limit.

If the theory is at a fixed point then there exists unitarity bounds which the scaling dimensions of gauge invariant operators should satisfy. If they do not satisfy this condition then either 1) the theory is not at a fixed point or 2) the operator is not part of the spectrum and has decoupled. Since $\operatorname{Tr} F^{2}$ is a spinless operator the unitarity bound is $D_{F^{2}}>1$ [32] which implies that $\gamma_{F^{2}}<3$. This condition is severely violated by many orders of magnitude in the entire range of $N_{f}$ where the theory is non-asymptotically free $N_{f}>\frac{11 C_{A}}{4 T_{r}}$. To see this, we note that $\delta \sim 1$ already for $N_{f} \sim \mathcal{O}\left(C_{A} / T_{r}\right)$.

For explicit numbers, consider for example fundamental fermions, $N_{c}=3$ and $N_{f}=17$. Then we find $\gamma_{F^{2}} \sim 10^{11}$ and increasing exponentially at larger $N_{f}$. For fundamental fermions, $N_{c}=2$ and $N_{f}=12$ we find similarly $\gamma_{F^{2}} \sim 10^{12}$. For higher representations this becomes even more dire: for adjoint fermions, $N_{f}=3$ and any $N_{c}$ we find $\gamma_{F^{2}} \sim 10^{13}$ and increasing exponentially at larger $N_{f}$.

The perturbative expansion we are using is of course expected to work only in a subrange of this interval. For fundamental fermions this range was estimated in [3] to be 
for $N_{f} \gtrsim 10 N_{c}$ while for adjoint fermions this range was estimated in [9] to be for $N_{f} \gtrsim 7$ for any $N_{c}$. These are the approximate ranges where the UV fixed point is conjectured to exist and here the value of $\gamma_{F^{2}}$ is exponentially large. With these considerations in mind we therefore conclude that if the UV fixed point exists then the glueball operator is not part of the spectrum of operators.

The fact that the glueball operator is not part of the spectrum might not be so odd after all. Remember that we are taking the limit of a large number of fermions but keeping the number of gluons fixed and finite. So in some sense the theory is flooded with fermions and the fact that the glueball operator then disappears from the spectrum may seem natural. We note that this should make for interesting lattice simulations to hopefully appear in the near future.

We note that an alternative interpretation of the violation of the unitarity of the anomalous dimension we have described above is that the theory is not at a fixed point. However, in that case one might expect that other observables would display similar inconsistency. We will now turn to the anomalous dimension of the spin- $1 / 2$ baryon operators, and show that they remain compatible with the existence of a fixed point.

Remember, that we are considering two different possibilities for the scheme dependent functions $s_{ \pm}$. Evaluating the anomalous dimension at $A_{\mathrm{UV}}=3-\delta$ for the first possibility $s_{ \pm}=1$ we find

$$
\gamma_{ \pm}=\frac{C_{r}(3-\delta)\left(27+3 \delta-2 \delta^{2}\right) \Gamma\left(2+\frac{2 \delta}{3}\right)}{72 T_{r} \Gamma\left(2-\frac{\delta}{3}\right) \Gamma\left(1+\frac{\delta}{3}\right)^{2} \Gamma\left(2+\frac{\delta}{3}\right)} \frac{1}{N_{f}} \sim \frac{9 C_{r}}{8 T_{r}} \frac{1}{N_{f}}+\frac{C_{r}}{2 T_{r}} \frac{1}{N_{f}} \delta+\mathcal{O}\left(\delta^{2}\right)
$$

while for the second possibility $s_{ \pm}=\left(1-\frac{2}{9} A\right)\left(1-\frac{1}{6} A\right)$ we find

$$
\gamma_{ \pm}=\frac{C_{r}(3-\delta)(6-\delta)(3+2 \delta) \Gamma\left(2+\frac{2 \delta}{3}\right)}{108 T_{r} \Gamma\left(2-\frac{\delta}{3}\right) \Gamma\left(1+\frac{\delta}{3}\right)^{2} \Gamma\left(2+\frac{\delta}{3}\right)} \frac{1}{N_{f}} \sim \frac{C_{r}}{2 T_{r}} \frac{1}{N_{f}}+\frac{5 C_{r}}{12 T_{r}} \frac{1}{N_{f}} \delta+\mathcal{O}\left(\delta^{2}\right)
$$

For completeness, in figure 2 we show the anomalous dimension as a function of the number of flavors in the entire range $16.5<N_{f}<300$ where the theory is non-asymptotically free. We remind the reader that the theory is conjectured to develop a non-trivial UV fixed point in the region only in the range $N_{f} \gtrsim 30$. The upper blue solid curve is for $s_{ \pm}=1$ while the lower red solid curve is for $s_{ \pm}=\left(1-\frac{2}{9} A\right)\left(1-\frac{1}{6} A\right)$.

The value of $\gamma_{ \pm}$is mostly sensitive to the scheme dependent function $s_{ \pm}$in the lower reaches of $N_{f}$. At $N_{f}=30$ we find $\gamma_{ \pm} \sim 0.1$ for $s_{ \pm}=1$ and $\gamma_{ \pm} \sim 0.04$ for $s_{ \pm}=$ $\left(1-\frac{2}{9} A\right)\left(1-\frac{1}{6} A\right)$ wheres at $N_{f}=100$ we find $\gamma_{ \pm} \sim 0.03$ for $s_{ \pm}=1$ and $\gamma_{ \pm} \sim 0.013$ for $s_{ \pm}=\left(1-\frac{2}{9} A\right)\left(1-\frac{1}{6} A\right)$. However although there is this sensitivity we observe that $\gamma_{ \pm}$is quite small for throughout the entire range $N_{f} \geq 30$. So with the full scaling dimension being $D_{ \pm}=\frac{9}{2}-\gamma_{ \pm}$the spin- $1 / 2$ baryond operators appear to be irrelevant $\left(D_{ \pm}>4\right)$ for any $N_{f}$ where the theory is at the UV fixed point never becoming marginal $\left(D_{ \pm}=4\right)$ or relevant $\left(D_{ \pm}<4\right)$. The unitarity bound for a spin- $1 / 2$ baryon operator is $D_{ \pm}>\frac{3}{2}[32]$ which is of course also safely satisfied within the entire range $N_{f}>30$. 


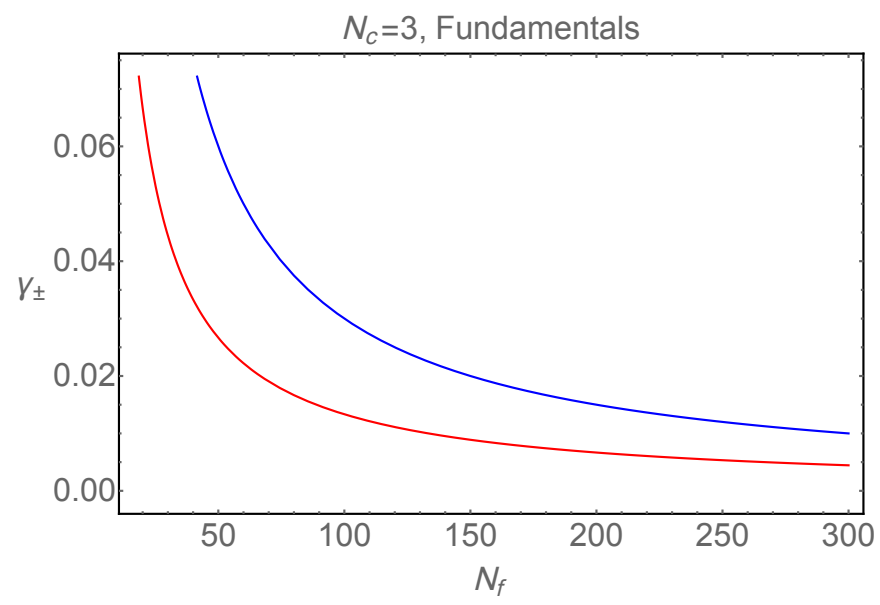

Figure 2. The anomalous dimension of the spin-1/2 baryons at the UV fixed point as a function of the number of flavors. The upper blue curve is for the choice $s_{ \pm}=1$ while the lower red curve is for the choice $s_{ \pm}=\left(1-\frac{2}{9} A\right)\left(1-\frac{1}{6} A\right)$.

\section{Conclusion}

In this paper we have considered the possibility of the emergence of UV fixed points in gauge theory at large number $N_{f}$ of fermionic matter fields. The beta function is known to leading order in $1 / N_{f}$ and here we have extended these results to determine the anomalous dimensions of the glueball and spin- $1 / 2$ baryon operators to this same order.

We evaluated the values of these anomalous dimensions at the UV fixed point. For the anomalous dimension of the baryon operators we found that their values are small and well within the unitarity bounds. On the other hand, for the glueball operator we found that the value of its anomalous dimension increases rapidly as $N_{f}$ increases leading to violation of the unitarity bound. While this result can be interpreted as an inconsistency undermining the existence of the UV fixed point and the validity of the large $N_{f}$ beta function, we pointed out that this effect can also arise from decoupling of the glueball operator from the spectrum at large $N_{f}$.

The research in this field is currently under active development, and our results should be useful in further analytic work as well as for possible lattice simulations of $\mathrm{SU}\left(N_{c}\right)$ gauge theories with many fermion flavors.

Finally and for completeness we add that although $\alpha \rightarrow 0$ as $N_{f} \rightarrow \infty$ it is $A$ that controls the dynamics and as we have seen $A_{\mathrm{UV}}$ is not necessarily small. So one could imagine that as the UV is approached the coupling $A$ increases to a sufficiently large value that triggers the formation of a fermion condensate which breaks chiral symmetry. Although mass terms are irrelevant as seen from the UV and hence one expects that the UV behavior should be independent of such symmetry breaking it would be interesting to study such scenarios in the future.

\section{Acknowledgments}

TAR wishes to thank N.A. Dondi for useful discussions and the University of Toronto for kind hospitality where the last part of the work was finished. This work is partially 
supported by the Danish National Research Foundation under the grant DNRF:90 and by the Academy of Finland project 310130.

\section{A Beta function coefficients}

In this appendix we give the perturbative formulas for the functions $H_{i}(A)$ appearing in eq. (2.3) inferred from the perturbative result for the beta function of generic gauge theory with gauge group $G$ and $N_{f}$ matter fields in representation $r$ of $G$. Validating these results requires the use of relations between the Euler $\Gamma(z)$, the digamma function $\psi(z)$, the polygamma function $\psi^{(m)}(z)$ and the Riemann zeta function $\zeta(z)$ which are provided in appendix B.

$$
\begin{aligned}
& H_{1}(A)=-\frac{11 C_{A}}{4 T_{r}}+\frac{5 C_{A}+3 C_{r}}{4 T_{r}} A-\frac{79 C_{A}+66 C_{r}}{288 T_{r}} A^{2}-\frac{53 C_{A}+154 C_{r}}{2592 T_{r}} A^{3} \\
& +\frac{\left(-229+480 \zeta_{3}\right) C_{A}+\left(214+288 \zeta_{3}\right) C_{r}}{20736 T_{r}} A^{4}+\ldots \\
& H_{2}(A)=-\frac{17 C_{A}^{2}}{8 T_{r}^{2}} A+\frac{1415 C_{A}^{2}+615 C_{A} C_{r}-54 C_{r}^{2}}{576 T_{r}^{2}} A^{2} \\
& -\frac{1}{10368 T_{r}^{4}}\left(3\left(3965+1008 \zeta_{3}\right) T_{r}^{2} C_{A}^{2}+32\left(268+189 \zeta_{3}\right) T_{r}^{2} C_{A} C_{r}+36\left(169-264 \zeta_{3}\right) T_{r}^{2} C_{r}^{2}\right. \\
& \left.+864\left(-11+24 \zeta_{3}\right) \frac{d_{r}^{a b c d} d_{r}^{a b c d}}{N_{A}}\right) A^{3} \\
& +\frac{1}{27648 T_{r}^{4}}\left(\left(6231+9736 \zeta_{3}-3024 \zeta_{4}-2880 \zeta_{5}\right) T_{r}^{2} C_{A}^{2}+16\left(46+1065 \zeta_{3}-378 \zeta_{4}\right) T_{r}^{2} C_{A} C_{r}\right. \\
& \left.+2\left(4961-11424 \zeta_{3}+4752 \zeta_{4}\right) T_{r}^{2} C_{r}^{2}+576\left(-55+123 \zeta_{3}-36 \zeta_{4}-60 \zeta_{5}\right) \frac{d_{r}^{a b c d} d_{r}^{a b c d}}{N_{A}}\right) A^{4}+\ldots \\
& H_{3}(A)=-\frac{2857 C_{A}^{3}}{1152 T_{r}^{3}} A^{2}+\frac{1}{20736 T_{r}^{4}}\left(-3\left(-39143-3672 \zeta_{3}\right) T_{r} C_{A}^{3}+\left(-7073+17712 \zeta_{3}\right) T_{r} C_{A}^{2} C_{r}\right. \\
& \left.+36\left(1051-264 \zeta_{3}\right) T_{r} C_{A} C_{r}^{2}-11178 T_{r} C_{r}^{3}+3456\left(-4+39 \zeta_{3}\right) \frac{d_{r}^{a b c d} d_{A}^{a b c d}}{N_{A}}\right) A^{3} \\
& \frac{1}{165888 T_{r}^{5}}\left(\left(-843067-332028 \zeta_{3}+16848 \zeta_{4}+356400 \zeta_{5}\right) T_{r}^{2} C_{A}^{3}\right. \\
& -3\left(5701+158712 \zeta_{3}-50976 \zeta_{4}+86400 \zeta_{5}\right) T_{r}^{2} C_{A}^{2} C_{r} \\
& -9\left(94749-57256 \zeta_{3}+20592 \zeta_{4}-79200 \zeta_{5}\right) T_{r}^{2} C_{A} C_{r}^{2}+108\left(2509+3216 \zeta_{3}-6960 \zeta_{5}\right) T_{r}^{2} C_{r}^{3} \\
& -864\left(-1347+2521 \zeta_{3}-396 \zeta_{4}+140 \zeta_{5}\right) C_{A} \frac{d_{r}^{a b c d} d_{r}^{a b c d}}{N_{A}} \\
& \left.-51840\left(13+16 \zeta_{3}-40 \zeta_{5}\right) C_{r} \frac{d_{r}^{a b c d} d_{r}^{a b c d}}{N_{A}}+1728\left(115-1255 \zeta_{3}+234 \zeta_{4}+40 \zeta_{5}\right) T_{r} \frac{d_{r}^{a b c d} d_{A}^{a b c d}}{N_{A}}\right) A^{4} \\
& +\ldots
\end{aligned}
$$




$$
\begin{aligned}
H_{4}(A)= & \frac{\left(-150653+2376 \zeta_{3}\right) C_{A}^{4}-864\left(-5+132 \zeta_{3}\right) \frac{d_{r}^{a b c d} d_{r}^{a b c d}}{N_{A}}}{41472 T_{r}^{4}} A^{3} \\
& +\frac{1}{20736 T_{r}^{5}}\left(3\left(39143-3672 \zeta_{3}\right) T_{r} C_{A}^{3}+\left(-7073+17712 \zeta_{3}\right) T_{r} C_{A}^{2} C_{r}+36\left(1051-264 \zeta_{3}\right) T_{r} C_{A} C_{r}^{2}\right. \\
& \left.-11178 T_{r} C_{r}^{3}+3456\left(-4+39 \zeta_{3}\right) \frac{d_{r}^{a b c d} d_{A}^{a b c d}}{N_{A}}\right) A^{4}+\ldots \\
H_{5}(A)= & \frac{\left(-8296235+78408 \zeta_{4}+451440 \zeta_{5}\right) C_{A}^{5}+2592\left(257-9358 \zeta_{3}+1452 \zeta_{4}+7700 \zeta_{5}\right) C_{A} \frac{d_{A}^{a b c d} d_{A}^{a b c d}}{N_{A}} A^{4}+\ldots}{1327104 T_{r}^{5}}
\end{aligned}
$$

\section{B The functions $\Gamma(z), \psi(z), \psi^{(m)}(z)$ and $\zeta(z)$}

The Euler gamma function $\Gamma(z)$, digamma function $\psi(z)$, polygamma function $\psi^{(m)}(z)$ and Riemann zeta function $\zeta(z)$ are defined as

$$
\begin{aligned}
\Gamma(z) & =\int_{0}^{\infty} x^{z-1} e^{-x} d x \\
\psi(z) & =\frac{d}{d z} \ln \Gamma(z)=\frac{\Gamma^{\prime}(z)}{\Gamma(z)}, \\
\psi^{(m)}(z) & =\frac{d^{m}}{d z^{m}} \psi(z)=\frac{d^{m+1}}{d z^{m+1}} \ln \Gamma(z), \quad \psi^{(0)}(z)=\psi(z), \\
\zeta(z) & =\frac{1}{\Gamma(z)} \int_{0}^{\infty} \frac{x^{z-1}}{e^{x}-1} d x-
\end{aligned}
$$

A first few examples which might be useful are

$$
\begin{aligned}
\Gamma(z+1) & =z \Gamma(z), \quad \psi(z+1)=\psi(z)+\frac{1}{z}, \quad \psi^{(m)}(z+1)=\psi^{(m)}(z)+\frac{(-1)^{m} m !}{z^{m+1}} \\
\Gamma(n) & =(n-1) !, \quad \psi(n)=H_{n-1}-\gamma, \quad \frac{\psi^{(m)}(n)}{(-1)^{m+1} m !}=\zeta(m+1)-\sum_{k=1}^{n-1} \frac{1}{k^{m+1}}
\end{aligned}
$$

where

$$
H_{n} \equiv \sum_{k=1}^{n} \frac{1}{k}, \quad \gamma \equiv-\Gamma^{\prime}(1)=-\psi(1)
$$

are the Harmonic numbers and the Euler-Mascheroni constant respectively.

Open Access. This article is distributed under the terms of the Creative Commons Attribution License (CC-BY 4.0), which permits any use, distribution and reproduction in any medium, provided the original author(s) and source are credited. 


\section{References}

[1] A. Palanques-Mestre and P. Pascual, The $1 / N_{F}$ Expansion of the $\gamma$ and $\beta$ functions in Q.E.D., Commun. Math. Phys. 95 (1984) 277 [InSPIRE].

[2] J.A. Gracey, The QCD $\beta$-function at $O\left(1 / N_{f}\right)$, Phys. Lett. B $373(1996) 178$ [hep-ph/9602214] [INSPIRE].

[3] B. Holdom, Large $N$ flavor $\beta$-functions: a recap, Phys. Lett. B 694 (2011) 74 [arXiv: 1006.2119] [INSPIRE].

[4] R. Shrock, Study of Possible Ultraviolet Zero of the $\beta$-function in Gauge Theories with Many Fermions, Phys. Rev. D 89 (2014) 045019 [arXiv:1311.5268] [INSPIRE].

[5] D.F. Litim and F. Sannino, Asymptotic safety guaranteed, JHEP 12 (2014) 178 [arXiv: 1406.2337] [INSPIRE].

[6] D.F. Litim, M. Mojaza and F. Sannino, Vacuum stability of asymptotically safe gauge-Yukawa theories, JHEP 01 (2016) 081 [arXiv: 1501.03061] [INSPIRE].

[7] O. Antipin, N.A. Dondi, F. Sannino, A.E. Thomsen and Z.-W. Wang, Gauge-Yukawa theories: $\beta$-functions at large $N_{f}$, Phys. Rev. D 98 (2018) 016003 [arXiv: 1803.09770] [INSPIRE].

[8] E. Mølgaard and F. Sannino, Asymptotically safe and free chiral theories with and without scalars, Phys. Rev. D 96 (2017) 056004 [arXiv:1610.03130] [INSPIRE].

[9] O. Antipin and F. Sannino, Conformal Window 2.0: The large $N_{f}$ safe story, Phys. Rev. D 97 (2018) 116007 [arXiv: 1709.02354] [INSPIRE].

[10] N.A. Dondi, G.V. Dunne, M. Reichert and F. Sannino, Analytic Coupling Structure of Large $N_{f}$ (Super) $Q E D$ and $Q C D$, arXiv:1903.02568 [INSPIRE].

[11] A.D. Bond and D.F. Litim, Theorems for Asymptotic Safety of Gauge Theories, Eur. Phys. J. C 77 (2017) 429 [Erratum ibid. C 77 (2017) 525] [arXiv:1608.00519] [InSPIRE].

[12] A.D. Bond and D.F. Litim, More asymptotic safety guaranteed, Phys. Rev. D 97 (2018) 085008 [arXiv: 1707.04217] [INSPIRE].

[13] A.D. Bond and D.F. Litim, Asymptotic safety guaranteed in supersymmetry, Phys. Rev. Lett. 119 (2017) 211601 [arXiv:1709.06953] [INSPIRE].

[14] A.D. Bond, D.F. Litim, G. Medina Vazquez and T. Steudtner, UV conformal window for asymptotic safety, Phys. Rev. D 97 (2018) 036019 [arXiv:1710.07615] [INSPIRE].

[15] D.F. Litim and M.J. Trott, Asymptotic safety of scalar field theories, Phys. Rev. D 98 (2018) 125006 [arXiv: 1810.01678] [INSPIRE].

[16] A.D. Bond and D.F. Litim, Price of Asymptotic Safety, arXiv: 1801.08527 [INSPIRE].

[17] J.K. Esbensen, T.A. Ryttov and F. Sannino, Quantum critical behavior of semisimple gauge theories, Phys. Rev. D 93 (2016) 045009 [arXiv:1512.04402] [INSPIRE].

[18] T. Alanne and S. Blasi, The $\beta$-function for Yukawa theory at large $N_{f}$, JHEP 08 (2018) 081 [Erratum JHEP 09 (2018) 165] [arXiv: 1806.06954] [INSPIRE].

[19] T. Alanne and S. Blasi, Abelian gauge-Yukawa $\beta$-functions at large $N_{f}$, Phys. Rev. D 98 (2018) 116004 [arXiv: 1808.03252] [INSPIRE].

[20] K. Kowalska and E.M. Sessolo, Gauge contribution to the $1 / N_{F}$ expansion of the Yukawa coupling $\beta$-function, JHEP 04 (2018) 027 [arXiv: 1712.06859] [INSPIRE]. 
[21] R. Mann, J. Meffe, F. Sannino, T. Steele, Z.-W. Wang and C. Zhang, Asymptotically Safe Standard Model via Vectorlike Fermions, Phys. Rev. Lett. 119 (2017) 261802 [arXiv: 1707.02942] [INSPIRE].

[22] J.A. Gracey, Large $N_{f}$ quantum field theory, Int. J. Mod. Phys. A 33 (2019) 1830032 [arXiv: 1812.05368] [INSPIRE].

[23] F. Sannino and K. Tuominen, Orientifold theory dynamics and symmetry breaking, Phys. Rev. D 71 (2005) 051901 [hep-ph/0405209] [INSPIRE].

[24] P.A. Baikov, K.G. Chetyrkin and J.H. Kühn, Five-Loop Running of the QCD coupling constant, Phys. Rev. Lett. 118 (2017) 082002 [arXiv: 1606. 08659] [INSPIRE].

[25] F. Herzog, B. Ruijl, T. Ueda, J.A.M. Vermaseren and A. Vogt, The five-loop $\beta$-function of Yang-Mills theory with fermions, JHEP 02 (2017) 090 [arXiv:1701.01404] [INSPIRE].

[26] L. Vecchi, Anomalous dimension of spin-1/2 baryons in many-flavor QCD, Phys. Rev. D 97 (2018) 116016 [arXiv:1607.02740] [INSPIRE].

[27] S. Krankl and A. Manashov, Two-loop renormalization of three-quark operators in QCD, Phys. Lett. B 703 (2011) 519 [arXiv:1107.3718] [INSPIRE].

[28] J.A. Gracey, Three loop renormalization of 3-quark operators in QCD, JHEP 09 (2012) 052 [arXiv: 1208.5619] [INSPIRE].

[29] M. Ciuchini, S.E. Derkachov, J.A. Gracey and A.N. Manashov, Computation of quark mass anomalous dimension at $O\left(1 / N_{f}^{2}\right)$ in quantum chromodynamics, Nucl. Phys. B 579 (2000) 56 [hep-ph/9912221] [INSPIRE].

[30] P.A. Baikov, K.G. Chetyrkin and J.H. Kühn, Quark Mass and Field Anomalous Dimensions to $\mathcal{O}\left(\alpha_{s}^{5}\right)$, JHEP 10 (2014) 076 [arXiv: 1402.6611] [INSPIRE].

[31] P.A. Baikov, K.G. Chetyrkin and J.H. Kühn, Five-loop fermion anomalous dimension for a general gauge group from four-loop massless propagators, JHEP 04 (2017) 119 [arXiv: 1702.01458] [INSPIRE].

[32] G. Mack, All unitary ray representations of the conformal group $\mathrm{SU}(2,2)$ with positive energy, Commun. Math. Phys. 55 (1977) 1 [inSPIRE]. 